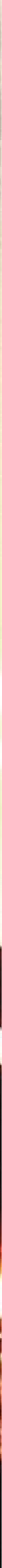




\section{Reto Verde: empresa y comunidad a favor del ambiente}

TAUG, en el marco del proyecto Reto Verde, promueve el diálogo entre comunidad y empresa sobre el cuidado del medioambiente a través del empoderamiento de los adolescentes de las comunidades cercanas. A la pardel proyecto, alienta el desa-

rrollo de habilidades como el liderazgo, la comunicación efectiva y el trabajo en equipo.

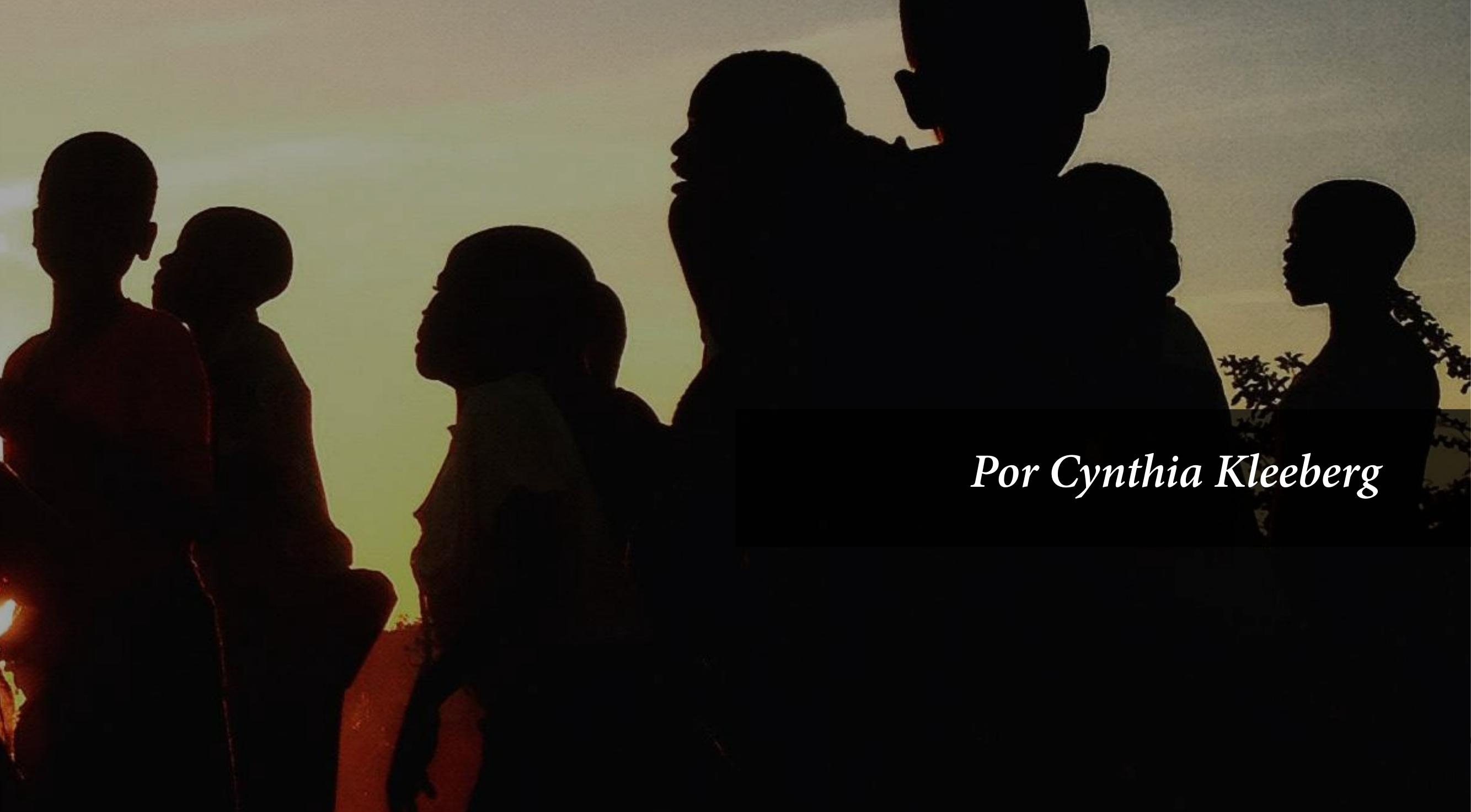


Ante las diferentes consecuencias sufridas por el cambio climático y aquellas que se advierte que tendrá este fenómeno, existe una acción global para mitigar y enfrentar estos perjuicios. En ese sentido, el Gobierno, los movimientos civiles, las empresas y todo tipo de organización están llamados a emprender prácticas que puedan alinearse a esta acción global. Una de las principales iniciativas es la de las Naciones Unidas, entidad que, a través de sus objetivos de desarrollo sostenible, señala la necesidad de optimizar el uso de recursos bajo una perspectiva ecoeficiente. Cabe resaltar también la importancia que ha tomado el Acuerdo de París en torno a los gases de efecto invernadero. Asimismo, en el Perú se pueden ver los compromisos adquiridos como nación y su estrategia de implementación en el PlanCC.

En este contexto, es importante que las empresas se vean inmersas en el cuidado del ambiente de manera proactiva, pues influencian a una cantidad importante de personas a lo largo de su cadena productiva. Este cuidado debe ser asumido como un compromiso con la sostenibilidad

\begin{tabular}{|c|c|}
\hline \multicolumn{2}{|c|}{ Cifras de avance 2014 - 2017} \\
\hline Número de kilos reciclados & $2086 \mathrm{Kg}$. \\
\hline Número de activaciones realizadas & 38 \\
\hline Número de jóvenes implicados en el proyecto & 118 \\
\hline Productos comunicacionales producidos & $\begin{array}{l}\text { * Videoclip iQue el planeta hable! } \\
\text { * Producto musical de hip hop enfocado } \\
\text { a la problemática de contaminación } \\
\text { medioambiental } \\
\text { * Videojuego ¡Que el planeta hable! } \\
\text { * Producto lúdico basado en la canción del } \\
\text { videoclip anterior }\end{array}$ \\
\hline $\begin{array}{l}\text { Número de actividades internas bajo } \\
\text { un esquema ecológico en HAUG }\end{array}$ & 6 \\
\hline
\end{tabular}

Elaboración propia

$\mathrm{y}$ con las futuras generaciones. Además, es una ventana de oportunidad para la propia empresa, pues puede evaluarse desde una mirada prospectiva; de esta manera, estará mirando al futuro y anticipando escenarios potenciales, lo que les dará un valor agregado en su planeamiento estratégico.

\section{HAUG y la sostenibilidad}

HAUG no ha sido ajeno a estos compromisos, en principio, porque es una empresa comprometida con la sostenibilidad desde el 2008, año en que se adhirió a los principios del Pacto Mundial de las Naciones
Unidas e inició su labor con la comunidad. Cabe mencionar que la empresa ha continuado con sus políticas de excelencia y sostenibilidad todos estos años y ha obtenido numerosos premios por sus prácticas innovadoras, por lo que ha llegado a figurar entre las empresas con excelente gestión de la responsabilidad social en el país. De hecho, durante estos últimos cuatro años, ha sido reconocida con el distintivo Empresa Socialmente Responsable (Distintivo $\mathrm{ESR}^{\circledR}$ ). De igual forma, da a conocer sus logros en materia de sostenibilidad mediante un reporte anual que es minuciosamente elaborado siguiendo la metodología G4 de GRI,

Tabla 2. Reconocimientos

Reconocimientos

* Invitación de la Municipalidad de Lurín para exponer el proyecto en el marco de la Semana del Medioambiente

* Adobe Youth Voices Awards (1.er puesto en la categoría Social Venture)

* Invitación a exponer el proyecto en el marco de la Feria EcoFest 2015, organizada por la Pontificia Universidad Católica del Perú

* Invitación a exponer el proyecto en el marco de la 1.a Feria de Responsabilidad Social de la Universidad Nacional de Ingeniería

* Invitación a programas radiales como Estoy verde y Pensando saludable

Elaboración propia 
y lo comparte de manera abierta con todos los stakeholders, lo que tiende puentes para el diálogo con cada uno de ellos.

Este compromiso de HAUG con el ambiente llevó a que su área de Sistemas de Gestión implemente una serie de controles de procedimientos a lo largo de su producción. Además, anualmente invierte en nueva tecnología para la mejora de su planta productiva. Estos esfuerzos la han llevado a obtener la certificación ISO 14001, referida a correctos procedimientos relacionados con el medioambiente.

\section{La experiencia del proyecto Reto Verde}

La iniciativa de HAUG a favor del medioambiente se plantea desde diversos frentes. A pesar de la importancia de trabajar el aspecto

Tabla 3. Los líderes comentan

\begin{tabular}{|l|l|}
\hline Los líderes comentan.. & $\begin{array}{l}\text { "El proyecto Reto Verde instruye a los } \\
\text { adolescentes en materia ambiental y } \\
\text { busca compartir sus conocimientos } \\
\text { mediante actividades lúdicas con } \\
\text { los colaboradores. De esta manera, } \\
\text { HAUG busca inculcar una cultura } \\
\text { de protección del medioambiente } \\
\text { que sea transversal a todos nuestros } \\
\text { stakeholders." }\end{array}$ \\
\hline Gamuel Yerusalimski \\
\hline GERENTE GENERAL DE HAUG
\end{tabular}

Elaboración propia procedimental y tecnológico, se hace necesario tomar en cuenta la dimensión humana de los colaboradores y grupos de interés, pues se busca motivar a cada una de las personas implicadas para que tomen decisiones asertivas en materia ambiental.

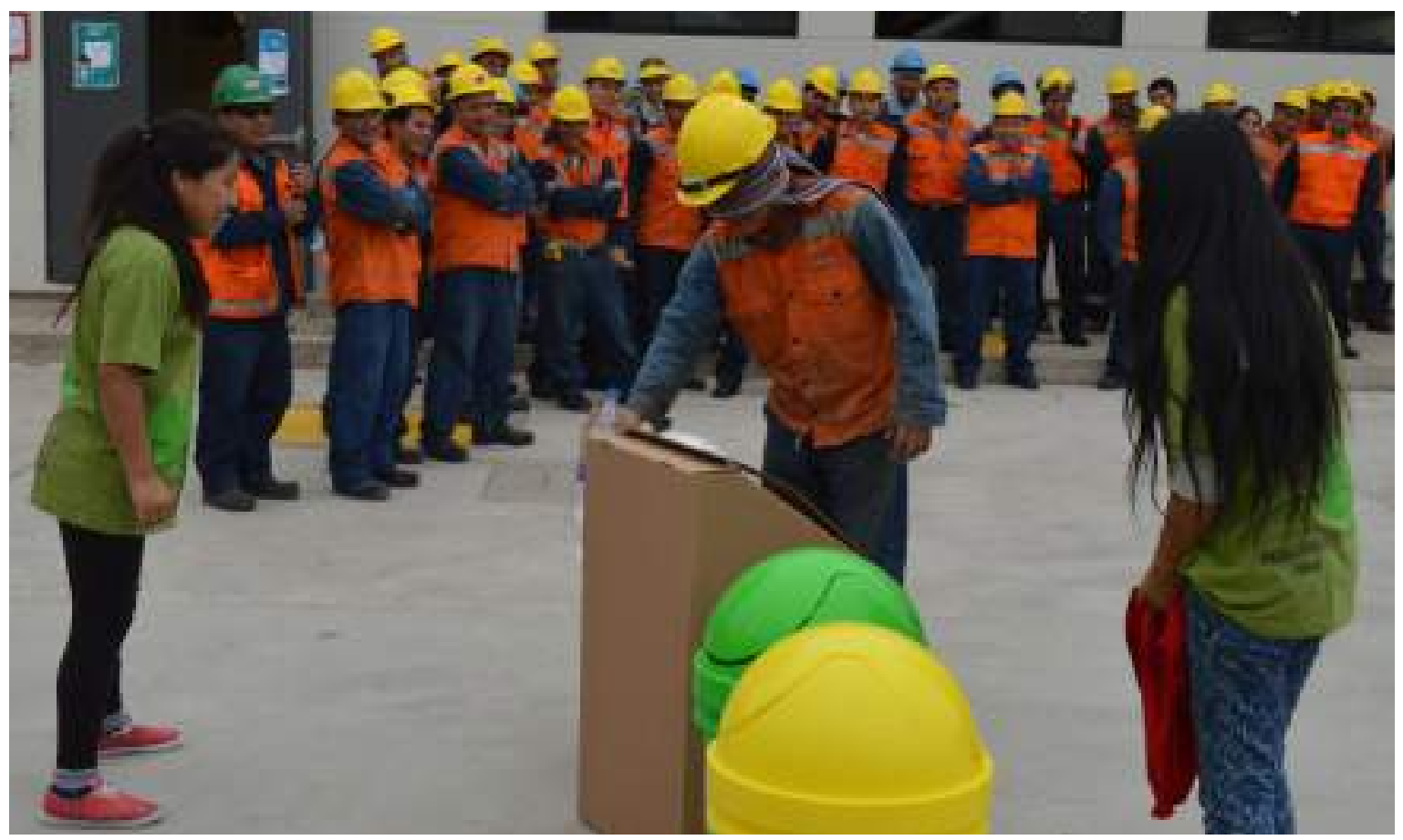

Figura 1. Aprendiendo la correcta disposición de los residuos sólidos Fuente: archivo fotográfico del Área de Responsabilidad Social de HAUG
De esta manera, HAUG, mediante su área de Responsabilidad Social, despliega una estrategia desde la cual se enfoca en la concientización de cada uno de sus colaboradores y los vecinos de su comunidad de manera motivadora, inclusiva e innovadora.
A partir del enfoque de valor compartido, se diseñó e implementó el proyecto Reto Verde. Este tiene por objetivo fomentar conductas ecoamigables en los vecinos de la comunidad y en los colaboradores. $\mathrm{Su}$ estrategia es la concientización, la práctica del reciclaje y el relacionamiento directo entre los grupos de interés de la empresa. Así también, se concentra en tres ejes:

- La educación ambiental. Se brindan clases de este tema dirigidas a jóvenes de la comunidad. Estas amplían su conocimiento sobre la materia a la vez que despiertan interés en las ciencias y el entorno que los rodea. Las clases son impartidas con metodologías lúdicas e innovadoras.

- La práctica manual y creativa. A partir del material reciclado tanto en la planta de Lurín como por los participantes mismos, se elaboran productos utilitarios, como cartucheras, portarretratos, lámparas, entre otros. 


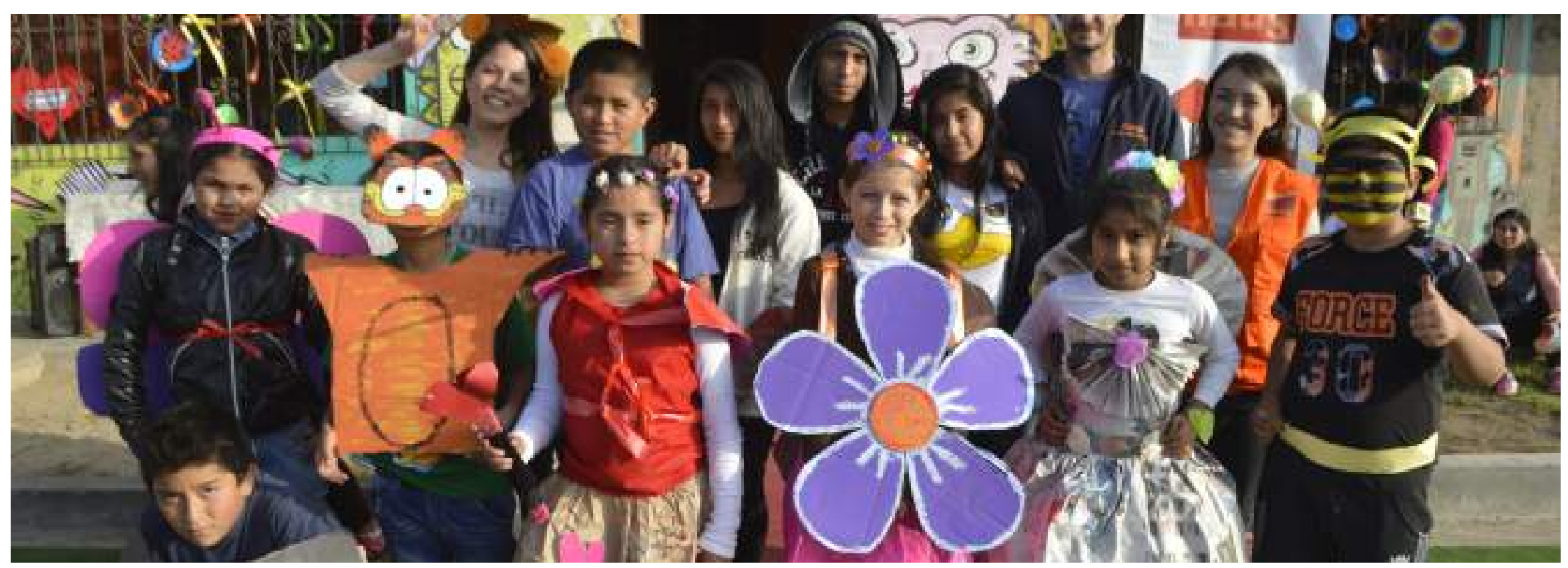

Figura 2. Concurso de disfraces con materiales reciclables

Fuente: archivo fotográfico del Área de Responsabilidad Social de HAUG

- La preparación de los jóvenes para que sean promotores de las prácticas ambientales. De esta forma aplican todo lo aprendido y desarrollan sus propios proyectos. Es entonces cuando los colaboradores de HAUG se vuelven su principal audiencia y sus principales seguidores.

Los jóvenes de la comunidad realizan activaciones en los espacios de HAUG y se dirigen a los colaboradores; ahí es donde ponen a prueba todos los conocimientos que adquirieron sobre consumo de energía, agua, transporte y disposición de residuos sólidos. Estas intervenciones son diseñadas e implementadas por ellos mismos, de modo que desarrollan habilidades como el liderazgo, la orientación al logro, el trabajo en equipo y la comunicación efectiva.

Es en el compartir de conocimientos, expectativas y motivaciones cuando se genera una atmósfera de cultura a favor del medioambiente, tanto en el público de la comunidad como en los colaboradores de HAUG, quienes reciben amigablemente a los jóvenes líderes comunitarios y participan de sus actividades. De esta forma se concientizan sobre la importancia del problema del cambio climático y de cómo ser parte de la solución.

Como se expuso en la introducción del artículo, el reto que pone el cambio climático requiere soluciones que partan de todos y cada uno de los actores, pues tanto las causas como sus consecuencias atañen a todos. A partir del proyecto Reto Verde, se ha querido replicar la necesidad de juntar esfuerzos de los grupos de interés que conciernen a HAUG en búsqueda de mejorar nuestras prácticas ambientales e impactar en el desarrollo del talento humano de los jóvenes de la comunidad.

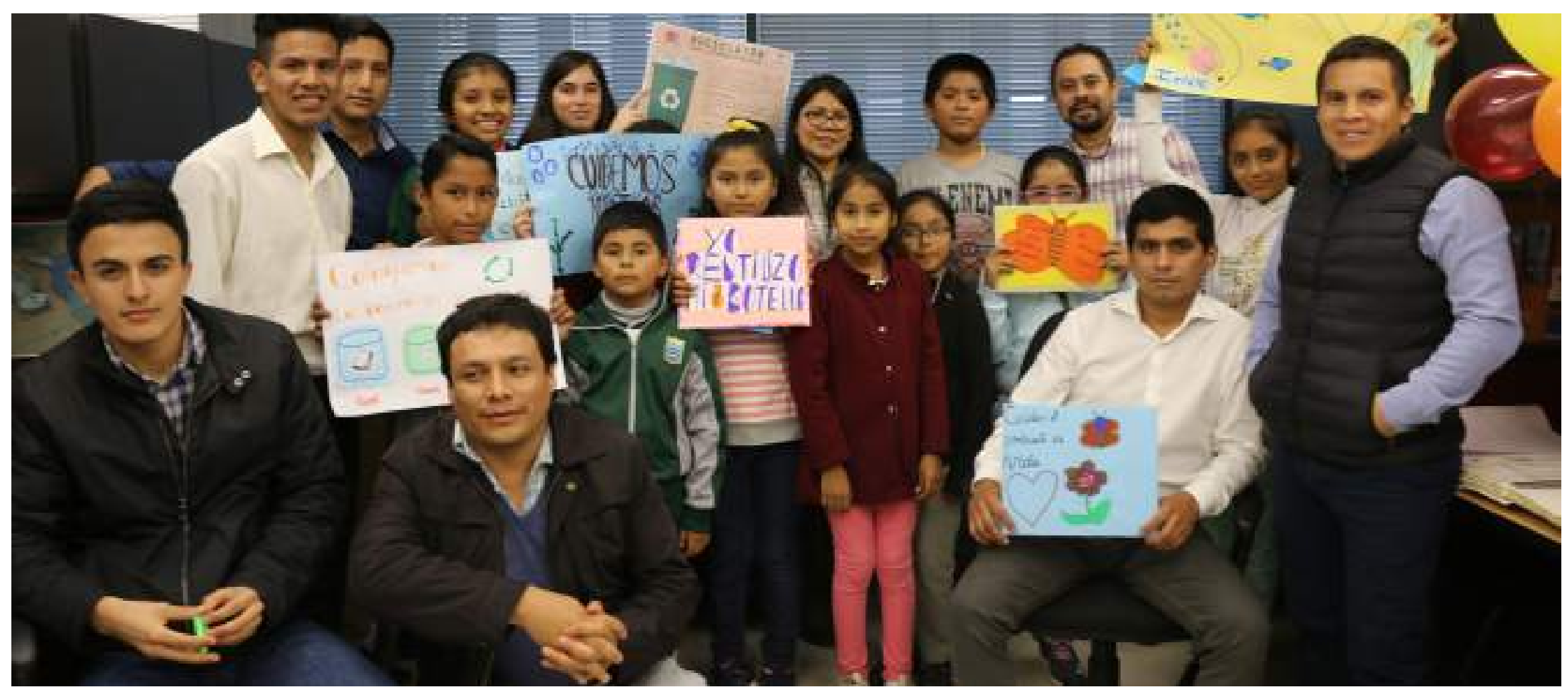

Figura 3. Cuidado de nuestros océanos. Activación en HAUG, Lurín

Fuente: archivo fotográfico del Área de Responsabilidad Social de HAUG 


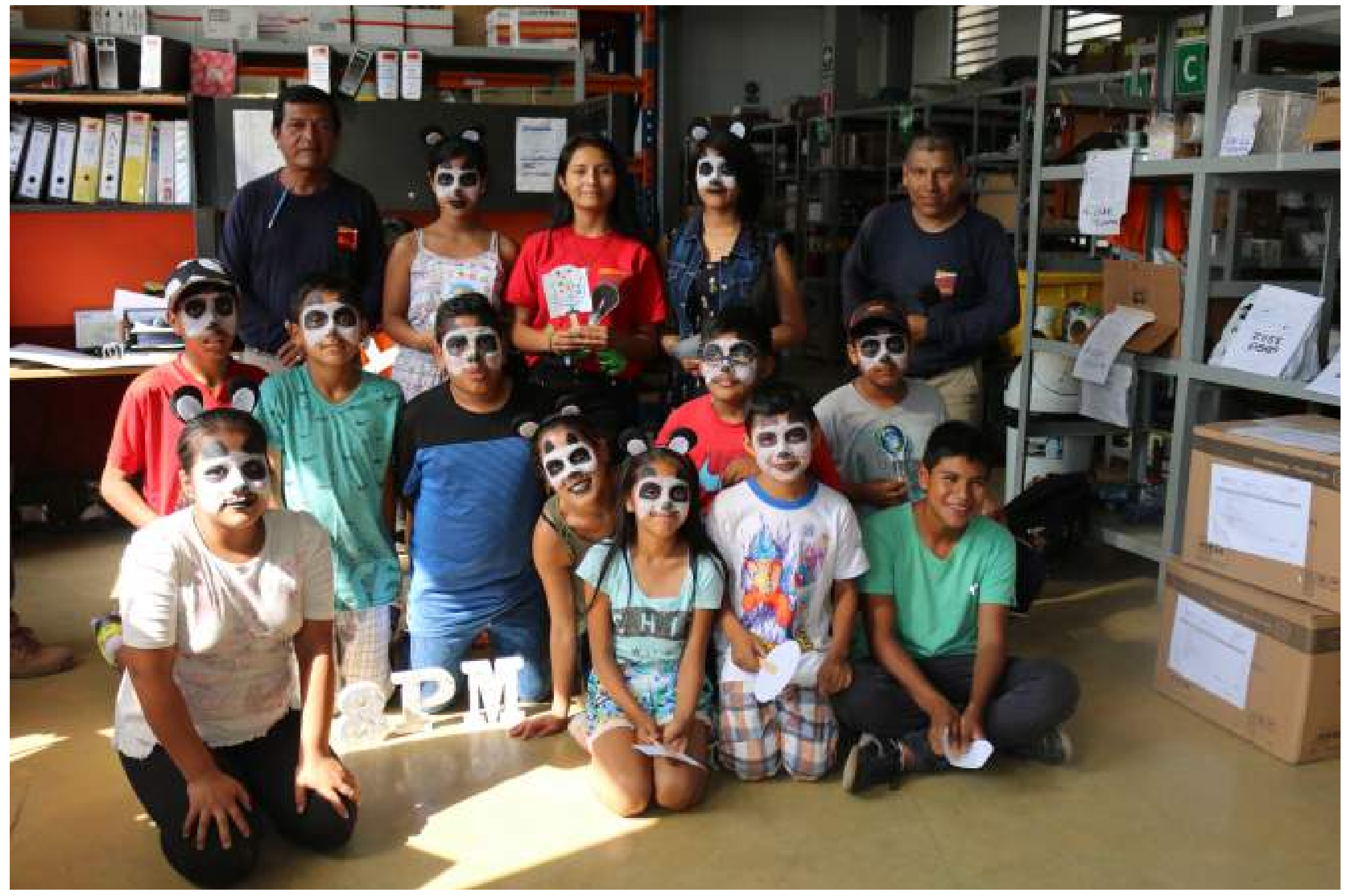

Figura 4. La hora del planeta. Activación en HAUG, Lurín

Fuente: archivo fotográfico del Área de Responsabilidad Social de HAUG

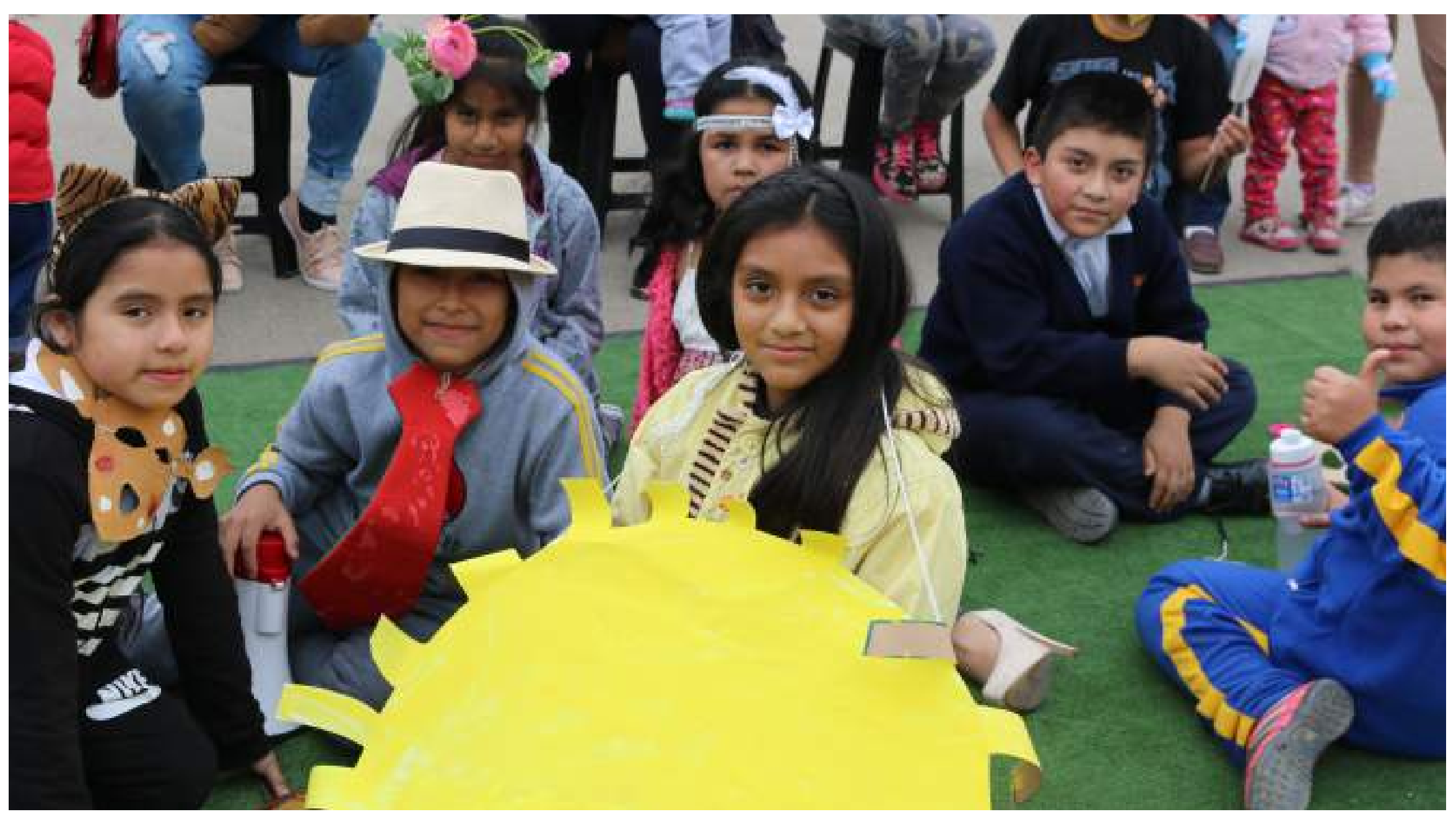

Figura 5. Teatro en la comunidad de Martha Milagros. La esperanza en el bosque

Fuente: archivo fotográfico del Área de Responsabilidad Social de HAUG 